\title{
Estado actual del mapeo linfático con biopsia del ganglio centinela en el melanoma cutáneo
}

\author{
José Francisco Gallegos-Hernández*
}

\begin{abstract}
Departamento de Tumores de Cabeza, Cuello y Piel, Hospital de Oncología, Centro Médico Nacional Siglo XXI, Instituto Mexicano del Seguro Social, Ciudad de México, México
\end{abstract}

Recibido el 10 de noviembre de 2016; aceptado el 25 de febrero de 2017

Disponible en Internet el 4 de agosto de 2017

La biopsia del ganglio centinela, procedimiento conocido como mapeo linfático con biopsia del ganglio centinela $(\text { MLBGC })^{1}$, fue desarrollada, descrita y publicada por Donald Morton y Alistair Cochran en $1992^{2}$. Está basada en los trabajos iniciales de Cabañas en pacientes con cáncer de pene y es el procedimiento estándar actual para la estadificación de los pacientes con melanoma cutáneo con Breslow mayor de $1 \mathrm{~mm}^{3}$ y sin ganglios palpables en la zona de drenaje linfático. Es uno de los avances más importantes en la cirugía oncológica en las últimas décadas; no solo acabó con la controversia que existía para decidir entre disección electiva y terapéutica en el melanoma con grosor intermedio, sino que su trascendencia ha sido tal que la técnica es ya fundamental para la estadificación ganglionar de muchas otras neoplasias, principalmente cáncer de mama, de piel no melanoma y de mucosas, y de cabeza y cuello ${ }^{3}$.

Es una técnica mínimamente invasiva, y aunque no exenta de complicaciones, obviamente estas son menos frecuentes en comparación con las que se presentan en pacientes sometidos a disección ganglionar electiva, de tal manera que permite, con menor tasa de compilaciones, clasificar por riesgo a los pacientes con melanoma cutáneo en etapas I y II según la presencia o ausencia de metástasis ganglionares subclínicas (previamente no identificadas).

Sin embargo, para que el MLBGC sea útil, cumpla su cometido y la estadificación sea real, requiere que se cumplan ciertos requisitos, al menos estos cinco:
- Cuidar los aspectos técnicos que permitan una alta tasa de identificación del ganglio centinela (GC) y una mínima tasa de falsos negativos.

- Una tasa de complicaciones baja.

- Una adecuada valoración histológica, incluida la inmunohistoquímica.

- Interpretar con precaución la sensibilidad del estudio.

- Una adecuada selección de los pacientes candidatos a ella, incluyendo el proceso diagnóstico.

La técnica con la cual se realiza el MLBGC es fundamen tal para asegurar una adecuada identificación del ganglio de primer relevo; sin duda, la técnica combinada (colorante yo radiocoloide) es el procedimiento estándar y debe realizarsè durante la resección del tumor primario. Si la lesión pigmentada ha sido previamente escindida, la biopsia no debe ser amplia y no tienen que hacerse reconstrucciones del área (colgajos o injertos).

Los objetivos perseguidos con una adecuada técnica de biopsia y de mapeo son obtener una alta tasa de identifica ción y una mínima de falsos negativos.

Actualmente, la tasa aceptada de identificación del GC es del $99.4 \%$, y la de falsos negativos depende del nivel de Breslow del tumor primario: $4.8 \%$ en pacientes con melanoma intermedio y $10.5 \%$ en pacientes con melanoma grueso, según los resultados obtenidos en el estudio MSLT-1 de Morton, et al. ${ }^{4}$.

*E-mail para correspondencia: jfgalh61@gmailcom (J.F. Gallegos-Hernández) 
Los puntos críticos a seguir para la adecuada identificación del verdadero GC son:

- La zona ganglionar de primer relevo no debe tener ganglios palpables.

- Es recomendable tener un ultrasonido que no muestre ganglios con sospecha de metástasis; si así fuese, deberá efectuarse biopsia por aspiración guiada por ultrasonido.

- Evitar biopsias con márgenes amplios, con injertos o con rotación de colgajos de cualquier índole.

- La utilización de coloide de Renio perilesional la víspera de la intervención o hasta 2 horas antes del procedimiento.

- La realización de gammagrafía preoperatoria (imagen bidimensional) que permite la localización anatómica (se puede hacer una identificación tridimensional con tomografía computarizada de emisión de fotón único, sobre todo en sitios de drenaje complejo, como la cabeza y el cuello) identificando sobre la piel el sitio de drenaje.

- La utilización de colorante perilesional al menos 10 minutos antes del procedimiento y ya realizada la incisión en el área linfoportadora.

- Efectuar palpación intraoperatoria del área para descartar la presencia de ganglios sospechosos al tacto que no hayan recibido el coloide ni el colorante (comunicación verbal del Dr. Merrick Ross).

Seguir estos pasos permite la tasa mas alta de identificación posible. La tasa de falsos negativos se minimiza siguiendo dichos pasos y aunando un adecuado estudio histopatológico que permita identificar metástasis submicroscópicas. Se recomienda evitar el estudio por congelación y esperar a la evaluación definitiva. Los GC negativos con el estudio convencional (tinción con hematoxilina y eosina) deben ser estudiados con tinciones de inmunohistoquímica (HMB-45 y/o Melan-A).

La probabilidad de tener un GC metastásico es del 5-40\%, y depende principalmente del nivel de Breslow. Aunque se han asociado otros factores, aún no están claramente definidos y son tema de controversia: el nivel de Clark, el sitio anatómico (mayor riesgo en melanomas de cabeza y cuello), la regresión del tumor primario, la ulceración (probablemente el segundo factor mas importante después del grosor tumoral), la invasión linfovascular, el índice mitótico (en los menores de $1 \mathrm{~mm}$ de Breslow) y la edad del paciente (con mayor frecuencia es metastásico en los menores de 20 años y raramente tienen metástasis en los mayores de 80 años) ${ }^{5}$.

Tanto en forma retrospectiva como recientemente en forma prospectiva ${ }^{4}$ se ha confirmado el valor pronóstico del GC, de tal manera que el estado histológico del GC es un factor independiente, como lo es el espesor tumoral. Así, en pacientes con melanomas con Breslow de $1.2 \mathrm{~mm}$ o mayores, la supervivencia es menor, y el intervalo libre de enfermedad es más corto en pacientes con GC metastásico en comparación con aquellos cuyo GC es negativo para metástasis.

Por otro lado, el valor terapéutico del GC es pobre; esto es, la posibilidad de que el MLBGC en los pacientes con GC metastásico impida la progresión neoplásica es cuestionable. En el estudio MSLT-1, la supervivencia no es mejor en los pacientes sometidos a biopsia del GC comparada con la de aquellos que quedaron en observación, y el intervalo li- bre de enfermedad solo es mejor $7 \%$ en melanomas intermedios y $10 \%$ en melanomas gruesos; esto es, los pacientes que se someten a MLBGC tienen un menor índice de recaída en la zona mapeada comparados con los que quedan en observación.

En pacientes con GC positivo para metástasis, el número de estas, así como el tamaño y la localización, son factores pronósticos para recurrencia y supervivencia.

Los pacientes con melanomas delgados, menores de 1 $\mathrm{mm}$ de Breslow, tienen muy poca posibilidad de metástasis en el GC, en promedio un 5.2\%, motivo por el que el MLBGC no está indicado. Sin embargo, existen subgrupos en los que debe considerarse el procedimiento. Probablemente el fac tor que predice la presencia de GC positivo en melanomas menores de $1 \mathrm{~mm}$ de espesor con mayor exactitud sea el espesor tumoral: la media de metástasis en el GC en pacientes con Breslow menor de $0.75 \mathrm{~mm}$ es del $2.9 \%$, y en los que tienen entre 0.75 y $1 \mathrm{~mm}$ es del $7.1 \%$.

Otros factores que influyen e incrementan la posibilidad de GC metastásico en pacientes con melanomas delgados son el índice mitótico mayor de una mitosis $/ \mathrm{mm}^{2}$, la invasión linfovascular, la ulceración (poco frecuente en los melanomas delgados), la infiltración tumoral linfocitaria y la regresión. Todos estos factores deben tomarse en cuenta cuando se tiene que decidir entre MLBGC o vigilancia; para algunos autores, la posibilidad de GC metastásico en presencia de at menos uno de los factores señalados alcanza el $18 \%$.

A diferencia de los melanomas intermedios, el pronóstico no cambia en relación al estado histológico del GC en los pacientes con melanoma menor de $1 \mathrm{~mm}$ de Breslow.

En los pacientes con metástasis en el GC, el consenso mundial es efectuar la linfadenectomía complementaria del área independientemente del tipo de metástasis, aunque en la actualidad sabemos que factores como el número de GC metastásicos, la presencia de metástasis parenquimatosas y no sub capsulares, la ulceración y el espesor tumoral se asocian en forma directamente proporcional a la posibilidad de ganglios no centinela metastásicos. Sin embargo, el valor de la lin fadenectomía complementaria por GC metastásico radica en que, en promedio, el $21 \%$ de estos pacientes tendrán ganglios metastásicos en la disección, lo que permite estratificarlos por riesgo de recurrencia; esto es, los que tienen metástasis en los ganglios no centinela tienen una peor supervivencia y un menor intervalo libre de enfermedad que aquellos con metástasis limitadas al GC, y eventualmente podrían ser can-o didatos a terapias adyuvantes o bien a ensayos clínicos.

En síntesis, el MLBGC se ha instituido como el abordaje estándar para la estadificación ganglionar en los pacientes con melanoma cutáneo con Breslow mayor de $1 \mathrm{~mm}$. Si no existe la posibilidad de realizarlo, pueden tomarse dos actitudes: vigilar la zona linfoportadora o remitir al paciente a centros o grupos que lo hagan apegados a la norma mundial (se prefiere esta actitud).

El MLBGC no está indicado en melanomas en etapa 0 , menores de $0.75 \mathrm{~mm}$ ni delgados entre 0.75 y $1 \mathrm{~mm}$, excepto si en el paciente coexisten otros factores de riesgo, aunque estos aún no están adecuadamente establecidos. La clave para obtener éxito es apegarse a la técnica recomendada desde el proceso diagnóstico.

Finalmente, la evidencia muestra que en los pacientes con GC metastásico la conducta a seguir es completar la linfadenectomía del área ganglionar sometida a mapeo. 


\section{BIBLIOGRAFÍA}

1. Gallegos JF. ¿Qué es el ganglio centinela? Concepto y aplicaciones en oncología. Acta Med Gpo Angeles. 2005;3:91-8.

2. Morton DL, Wen DR, Wong JH, et al. Technical details of intraoperative lymphatic zapping for early stage melanoma. Arch Surg. 1992;127:392-9.

3. Nieweg OE, Uren RF, Thompson JF. The history of sentinel node biopsy. Cancer J. 2015;21:3-6.
4. Morton DL, Thompson JF, Cochran AJ, et al. Final trial report of sentinel-node biopsy vs nodal observation in melanoma. New Engl J Med. 2014;370:599-609.

5. Balch CM, Thompson JF, Gershenwald JE. Age as a predictor of sentinel node metastasis among patients with localized melanoma: an inverse correlation of melanoma correlation and incidence of sentinel node metastasis among young and old patients. Ann Surg Oncol. 2014;21:1075-81. 